Intervention for problem drinking Kypri et al.

\title{
Innovative approaches to intervention for problem drinking
}

\author{
Kypros Kypri $^{\mathrm{a}}$ \\ Thiagarajan Sitharthan ${ }^{\mathrm{b}}$ \\ John A. Cunningham ${ }^{\mathrm{C}}$ \\ David J. Kavanagh ${ }^{\mathrm{d}}$ \\ Johanna I. Dean ${ }^{\mathrm{e}}$
}

${ }^{\text {a }}$ School of Medical Practice and Population Health, University of Newcastle, Australia

${ }^{\mathrm{b}}$ Department of Psychological Medicine, University of Sydney, Australia

${ }^{\mathrm{c}}$ Centre for Addiction and Mental Health, Ontario, Canada

${ }^{\mathrm{d}}$ Department of Psychiatry, University of Queensland, Australia

${ }^{\mathrm{e}}$ Research Psychologist, Newcastle, Australia

Correspondence to Kyp Kypri PhD, School of Medical Practice and Population Health, University of Newcastle, Locked Bag 10, Wallsend, NSW 2287, Australia Tel: +61 249275957

Fax: +61 249246208

E-mail: kypri@tpg.com.au

Purpose of review

To critique the recent literature on telephone, correspondence-based, and computerized interventions for alcohol problems, which enhance or substitute for practitioner-delivered treatments.

Recent findings

There is an unmet need for screening, assessment and intervention for alcohol problems, in part because of the difficulty in accessing such treatment within the current health care system. Research on the efficacy of correspondence or electronic (for example Internet-based) interventions is beginning to emerge. In the period 2003-2004 we identified nine acceptability or feasibility studies of these approaches and seven efficacy trials covering a wide range of settings. These modes of intervention are acceptable to patients and the public, and with careful planning, can be implemented in a variety of settings. Treatment trials demonstrate the efficacy of these interventions in reducing hazardous drinking by university students, in delaying initiation of heavy drinking in children and adolescents, and, intriguingly, in addressing insomnia among recovering alcoholics. 
Summary

There is strong support among potential users for alcohol interventions that employ telephone assistance, written correspondence, and the Internet. These new technologies offer the prospect of increasing the reach of interventions for problem drinking and being cost-effective alternatives or supplements to face-to-face health service delivery.

Keywords

alcohol; computer; correspondence; Internet; intervention; screening; telephone

\title{
Abbreviations
}

\author{
AUDIT Alcohol Use Disorders Identification Test \\ CBT cognitive behaviour therapy \\ e-SBI electronic screening and brief intervention \\ RCT randomized controlled trial \\ SBI screening and brief intervention
}

\section{Introduction}

There is growing interest in the use of telephone, correspondence-based and computerized interventions for alcohol problems. This is partly due to the scarcity of time for alcohol interventions within primary care settings. These new technologies may help to overcome some patients' reluctance to discuss their alcohol consumption, as well as ensuring consistent delivery of interventions, and improving access to preventive health care. There is potential to employ these technologies outside the health care system and to extend the reach of interventions to people with undiagnosed or untreated alcohol use disorders. In this paper we review the evidence for these alternative intervention methods for alcohol problems, when used independently or in conjunction with practitioner-delivered therapies. The full spectrum of alcohol problems from hazardous drinking to alcohol dependence is encompassed.

\section{Screening and brief intervention for primary care patients}

A significant advance in the treatment of hazardous alcohol consumption and thereby the prevention of alcohol-related harm over the last two decades has been the development and evaluation of screening and brief intervention (SBI). SBI typically involves opportunistic administration by a GP or nurse of a brief screening questionnaire such as the Alcohol Use Disorders Identification Test (AUDIT) [1] and, for those who screen positive, provision of 5-10 min of brief advice or a short session ( $<30$ min) of motivational therapy [2]. For people identified with severe problems or an established alcohol dependence, a referral may be made for further assessment and specialist treatment [3]. More than 40 randomized controlled trials (RCTs) have been published on SBI, most of which have been in primary care settings.

In 2002, Moyer and colleagues [4] published a pivotal metaanalysis which revealed significant, albeit modest, reductions in hazardous drinking lasting at least 6- 
12 months among people who were not specifically seeking treatment. Among those actually seeking treatment, the effects of SBI were similar to those of more intensive interventions [4]. On the basis of an extensive review of the effectiveness of SBI in primary care [5], the US Preventive Services Task Force $\left[6^{\circ}\right]$ found that SBI of $15 \%$ min is helpful, and that multi-contact interventions are effective for patients ranging from 17 to 70 years of age.

\section{The need for innovative intervention approaches}

Recent evidence reveals extensive unmet need for advice about alcohol problems among primary care patients. On the basis of a telephone survey across 10 states in the USA, Denny et al. [ $\left.7^{\circ}\right]$ estimated that only $23 \%$ of binge drinkers, who had received a routine medical check-up in the previous year, reported that the doctor had discussed their drinking. Discussion was less likely for women than men, and for white than black patients. Research from Finland also reveals low rates of systematic screening and intervention by GPs [8]. Notably, Aalto and Seppa [9] observed that in the rare instances when GPs discussed alcohol, 81\% of patients considered this helpful.

Although evidence concerning the efficacy of SBI for patients with alcohol problems (excluding those with alcohol dependence) is strong, it is rarely offered in primary care. This is not explained by lack of staff knowledge or skill. Beich and colleagues [10] reported that many GPs are reluctant to initiate discussion about alcohol with patients through fear of damaging rapport. Lock and her colleagues [11] found that many medical practice staff do not see screening as a legitimate part of their role.

McCambridge and colleagues [12] examined the effect of a motivational intervention to encourage GP delivery of SBI in an uncontrolled study. There was no evidence of significant change in willingness to use SBI or in the detection of alcohol problems. However, high baseline rates of reported use of alcohol questions and opportunistic intervention made it difficult to demonstrate increases in these behaviours or related intention to change.

Even if practitioners show willingness to undertake training, it does not guarantee

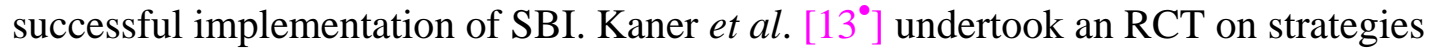
to increase the use of SBI by nurses, randomizing 212 practices. Three methods were compared: written guidelines only (control), 30\% min of training, and training plus biweekly telephone support. Rates of participation were high in all conditions ( $\geq 70 \%$ of eligible practitioners). Training resulted in more nurses implementing SBI, and more patients per practice screened and given SBI for at-risk drinking. However, more patients who had no alcohol problems received SBI by nurses who had been trained in these techniques, and so cost-benefit results were comparable across the three groups. Furthermore, most nurses delivered intervention to no more than a single patient and there remained high variability in the degree to which SBI was applied.

Even if all primary care practitioners do routinely implement SBI, some patients have little or no contact with primary care settings, or are reluctant to acknowledge 
problems in a face-to-face interaction. There is a need, therefore, for alternative methods of treatment delivery.

\section{The evidence base for innovative interventions}

Many websites offer treatment or information to people who are concerned about their drinking, but compelling evidence to support their effectiveness is hard to find. On the basis of an extensive search, Toll et al. [14 $\left.{ }^{\circ}\right]$ presented a description of alcohol-related websites, reporting that only five of 68 treatment-related sites (7\%) provided on-line treatment. Seventy percent of treatment sites applied 12-step facilitation, and only 4\% focused on motivational strategies. Few sites provided references $(13 \%)$ or outcome data $(7 \%)$ on the treatment that they recommended.

A qualitative review by Copeland and Martin [15 ${ }^{\circ}$ identified five papers on webbased interventions for alcohol misuse, but only one controlled trial. They concluded that web-based interventions have a potentially wide reach and are generally well accepted by users, but that there is insufficient evidence as yet on their efficacy.

In a literature search conducted by the authors of the present review, nine acceptability or feasibility studies were identified, together with seven efficacy trials.

\subsection{The acceptability of electronic SBI in health care settings}

Six studies examining the acceptability of electronic SBI (e-SBI) in health care settings were identified. The settings studied included an emergency department, a university student health service, a specialist treatment service, a sexual health clinic, and a general medical centre.

The emergency department has often been identified as a setting that offers a window of opportunity for alcohol interventions. Given the high injury risk with alcohol involvement among young people, the study by Gregor et al. $\left[16^{\circ \bullet}\right]$ is of particular interest. Injured patients $(n=843)$ aged $14-18$ years were invited to complete an interactive alcohol intervention on a laptop computer (78\% completed). Of patients who reported recent alcohol use, $74 \%$ said the program made them reconsider their drinking. The study shows that the use of e-SBI in emergency medicine is feasible. Previous research suggests that such interventions can be effective in reducing hazardous consumption in this age group [17], but replication of these findings is required.

In a web-based survey of a random sample of 1564 university students (82\% response), Kypri and colleagues [ $\left[18^{\circ}\right]$ sought to gauge hazardous drinkers' interest in a range of primary care-based services aimed at reducing alcohol-related problems, including standard health education, web-based assessment and personalised feedback (e-SBI), and brief intervention administered by a doctor, nurse, or counsellor. Respondents were asked whether they would use each service if they had a drinking problem. Table 1 Table 1 shows that e-SBI had significantly greater support (82\%) among hazardous drinkers than did health education seminars (40\%) or practitionerdelivered interventions (58\%). 
Working in a specialized treatment centre, Squires and Hester [19] employed the 'Drinkers Check-Up', a computer-presented, stand-alone interactive intervention for problem drinking. The intervention is based on motivational enhancement therapy and consists of assessment and personalized feedback, with advice tailored to the user's readiness to change. The acceptability of the intervention was assessed in three users of the program in a clinical setting. Two reported large reductions in consumption 12 months after intervention, and all reported that the program motivated them to seek further assistance.

Given the increased risk of unprotected sex attributable to hazardous drinking, the sexual health clinic would seem to be an appropriate venue for brief opportunistic intervention. In this setting, Crawford et al. [20] sought to determine whether patients would be receptive to information regarding their alcohol use. Patients were screened and those drinking excessively were given a self-help leaflet or an appointment to see an alcohol health worker. More patients accepted the self-help leaflet than the appointment. Importantly, of those asked to see an alcohol health worker, only one attended.

Primary health care settings, such as general practice, would seem to offer considerable opportunities for intervention, but as reported earlier, this is not translated into routine practice. Methods that can reduce reliance on GP time and are appealing to patients have considerable potential. Gerbert et al. [21 $\left.{ }^{\circ}\right]$ sought to assist busy physicians in identifying and treating alcohol disorders. Patients were invited to interact with a 'video doctor', a program operated from a laptop computer. Following a brief assessment of drinking status, the video doctor provided a range of tailor-made motivation enhancing statements to promote behavioural change. The program was well accepted by patients and may be easily disseminated, but its efficacy in reducing drinking was not reported.

\subsection{The acceptability of SBI in the general population}

Wild and colleagues $\left[22^{\circ}\right]$ utilized a telephone survey (65\% response) with a random sample of residents in Alberta, Canada to gauge interest in self-help materials for problem drinking. Of 7833 current drinkers who had not received treatment for an alcohol disorder, $22 \%$ were interested in receiving a self-help pamphlet 'that would help people decide whether or not they would like to reduce their drinking' (p. 129). Generalized to the population as a whole, this represents a large number of people who may be willing to accept an inexpensive intervention. Self-help materials may also form part of stepped care, when people who require additional assistance might be encouraged to seek subsequent face-to-face intervention.

Several studies have examined the development of various types of e-SBI available to Internet users. These sites attracted a variety of age groups via several recruitment mechanisms. Lieberman [23] reported on a web survey of 1207 'alcohol abusers' who accessed a site providing e-SBI (http://www.alcoholcheckup.com) over a 25-month period. Half (53\%) were men, and their mean age was 35. Respondents who reported readiness to change their drinking tended to find the instrument most useful, and the 
provision of new information was also associated with higher rated utility (e.g. comparison of respondents' drinking with national norms).

Linke et al. [24] studied individuals accessing a website offering a 6-week program including elements of motivational enhancement therapy and cognitive behaviour therapy (CBT). Visitors could complete a screening questionnaire, and those who tested positive were encouraged to register for the program. Of 7581 visits to the site over 6 months, there were 1319 registrations from 41 countries, although only $6 \%$ of those completed the program. Over half of users were male (56\%) and $77 \%$ were white. Users found the site via search engines (35\%), links from other sites (18\%), and newspapers or magazines (26\%).

An impressively large study was conducted by Saitz et al. [25 $5^{\circ 0}$, who examined use of a site offering e-SBI, which received 115925 visits per year. Over 14 months, screening questionnaires were completed by $39 \% 482$ adults (66\% male). Respondents were typically aged $18-24$ years (30\%) or 25-34 years (33\%), with few (1\%) 65 or older. Two-thirds (65\%) screened positive for hazardous drinking on the AUDIT. A third of users visited a segment offering further help or referral for treatment. The study showed that a well-designed and resourced program can reach large numbers of hazardous drinkers, and facilitate referral for further care.

The findings of the last three studies in this section should be interpreted in light of the highly self-selected samples: it may be less likely that people would complete surveys if they found the materials unhelpful.

\subsection{Controlled trials of the efficacy of intervention}

Trials published during the review period cover a broad range of settings, including primary care $\left[26^{\bullet \bullet}, 27^{\circ}\right]$, specialist services $\left[28^{\circ}\right]$, a workplace $\left[29^{\circ}\right]$, and the wider community $\left[30^{\circ \bullet}\right]$. Two were conducted in laboratories, with psychology students $\left[31^{\bullet}, 32^{\circ}\right]$. Three studies $\left[26^{\bullet \bullet}, 30^{\bullet \bullet}, 32^{\circ}\right]$ used computers and the remainder used written correspondence or telephone support.

\subsubsection{Interventions in primary care}

Despite considerable interest in e-SBI as a concept, and some promising developmental studies (e.g. [33]), RCTs of this intervention have appeared only recently. Kypri and colleagues $\left[26^{\circ}\right]$ invited patients attending a primary care facility within a university to complete a 3 min computerized questionnaire on their drinking, including the AUDIT. Those screening positive were randomly assigned to a further 10-15 min of computerized assessment and personalized feedback or to a leafletonly control condition. Assent to screening was high (93\%). Assessments 6 weeks and 6 months later showed reductions of $20-30 \%$ in consumption and alcohol-related problems in the intervention group relative to controls. Although of modest size $(n=104)$, this study had particularly low attrition (10\% at 6 months), an unusually high initial participation rate, and it was conducted in a naturalistic setting: a busy primary care facility.

Curry et al. $\left[27^{\circ}\right]$ investigated the impact of physician counselling with and without telephone support. Patients who received counselling plus telephone support fared 
better than controls. However, compared with similar opportunistic intervention programs, initial consumption was low, averaging 14 drinks per week, with only 5\% consuming four or more drinks per day. Although three telephone calls per patient were scheduled, most participants accepted only one call from the counsellor. Of those receiving the self-help manual, 61\% claimed to have read at least part of it, and $38 \%$ found it useful. More patients from the intervention group dropped out, and the authors ascribed this to stigma associated with the study's focus on alcohol use.

\subsubsection{Interventions in specialist services}

Insomnia is commonly observed among alcohol-dependent patients, even a considerable period of time after alcohol cessation. In a three-arm RCT by Currie et al. [28 ${ }^{\circ \bullet}$, volunteer patients were assigned to five sessions of individual CBT, a selfhelp manual plus five brief phone calls, or a wait-list control. Improvements in sleep were observed among treated patients, although there was no evidence that the intervention prevented relapse to drinking. At 6-month follow-up, $60 \%$ of those treated had discontinued use of sedative medications. This study provides a compelling case for the use of self-help materials with telephone support to help problem drinkers sleep better without medication. The study supplements existing evidence supporting the benefits of correspondence-based interventions for alcohol misuse itself (e.g. [34]).

\subsubsection{Workplace interventions}

Occupational settings have shown mixed results on the feasibility and efficacy of SBI (e.g. [35]). Walters and Woodall [29 $\left.{ }^{\circ}\right]$ studied 48 employees of a manufacturing firm who volunteered to receive a free confidential assessment of their drinking by mail. On the basis of screening, respondents were randomly assigned to mailed personalized feedback or a waiting list control group, with 8 and 16-week follow-up assessments. The intervention group decreased their consumption significantly relative to controls. The study is limited by the fact that most participants were moderate drinkers (averaging seven drinks per week) and only $7 \%$ of potential respondents participated.

\subsubsection{Interventions in community settings}

In contrast to the studies so far described, which examine secondary prevention approaches, the study by Schinke et al. $\left[30^{\circ \bullet}\right]$ examined a primary prevention approach. It is unique and impressive in terms of its setting, the age of the target group, and the extent of follow-up. Using 46 community agencies offering social services, and recreational or after-school programs, 514 urban 10-12-year-olds were recruited (51\% girls). Respondents in each site were randomized to either a CD-ROM intervention, a CD-ROM intervention plus parent intervention, or to a control group. Follow-up was conducted at 1, 2 and 3 years following intervention. Booster sessions were provided between each follow-up. The CD-ROM intervention consisted of ten 45.min sessions providing instruction and problem-solving skills to help young people avoid alcohol. The parent intervention provided information on goals of the youth intervention and demonstrated how parents could help their children apply the intervention. Information on family involvement, peer influence and recent use of alcohol was collected at each follow-up. Respondents in the intervention conditions reported lower alcohol use and less initiation to heavy drinking at 1, 2 and 3-year follow-ups compared with controls. Respondents in the CD-ROM plus parent 
intervention reported less increased use than those in the CD-ROM only condition. While a relatively intensive intervention, the study is of interest because of the difficulties usually faced in effecting change in this age group [36].

\subsubsection{Laboratory studies}

Two studies report the effects of interventions conducted in undergraduate psychology classes. Murphy et al. [31 $\left.{ }^{\circ}\right]$ recruited 54 heavy drinkers through a screening survey offered for course credit. Participants were randomized to receive personal drinking feedback only or personal drinking feedback plus motivational interview. Participants in the first condition were asked to review the feedback and an advice sheet for 30 min. Those in the second condition reviewed the feedback for 30 min with a clinician who employed motivational interviewing. At 6-month follow-up, there was evidence of a reduction in drinking in both groups, but differences between groups were nonsignificant.

Neighbors et al. [32 ${ }^{\circ}$ randomized 252 heavy drinkers to computerized feedback comparing individual consumption and perceived drinking norms to actual norms (eSBI), or to a no intervention control group. Participants receiving e-SBI reported significantly greater reductions in drinking at 3 and 6-month follow-up compared with controls. Mediator analyses provided support for the hypothesis that normative feedback has an impact because it alters respondents' perceived drinking norms. Some support was also found for the moderator hypothesis that normative feedback would be more important for respondents who drink for social reasons.

\section{Conclusions}

This literature review demonstrates strong interest in innovative methods of alcohol intervention to supplement standard treatment in various health care settings or to provide stand-alone intervention in the wider community. There were few efficacy trials over the period, despite the lead given by the positive findings of previous trials (e.g. [34,37]). Correspondence-based interventions and telephone support studies had

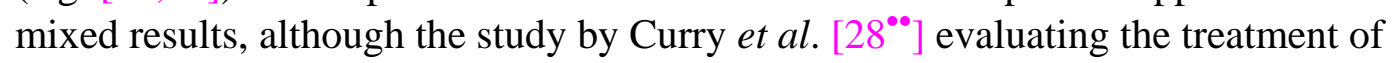
insomnia with self-help materials and telephone support is promising.

The three efficacy trials on computerized interventions $\left[26^{\circ \bullet}, 30^{\circ \bullet}, 32^{\circ}\right]$ indicate that these approaches can reduce hazardous alcohol consumption. Results showing delays in initiation to heavy drinking among younger adolescents receiving a CD-ROM intervention are particularly encouraging $\left[30^{\circ}\right]$. The evaluation of innovative interventions for alcohol problems is still at a fledgling stage. Existing studies need replication, and effort must be made to conduct controlled trials in naturalistic conditions to ensure generalizability of the findings to routine healthcare delivery.

\section{References}

1 Saunders JB, Aasland OG, Babor TF, et al. Development of the Alcohol Use Disorders Identification Test (AUDIT): World Health Organisation Collaborative Project on Early Detection of Persons with Harmful Alcohol Consumption - II. Addiction 1993; 88:791-804.

2 Miller WR, Rollnick S. Motivational interviewing: preparing people to change addictive behavior. New York: Guilford Press; 1991. pp. xvii, 348. 
3 Saunders JB, Lee NK. Hazardous alcohol use: its delineation as a subthreshold disorder, and approaches to its diagnosis and management. Compr Psychiatry 2000; 41 (2 Suppl 1):95-103.

4 Moyer A, Finney JW, Swearingen CE, Vergun P. Brief interventions for alcohol problems: a meta-analytic review of controlled investigations in treatmentseeking and non-treatment-seeking populations. Addiction 2002; 97:279-292.

5 Whitlock EP, Polen MR, Green CA, et al. Behavioral counseling interventions in primary care to reduce risky/harmful alcohol use by adults: a summary of the evidence for the U.S. Preventive Services Task Force. Ann Intern Med 2004; 140:557-568.

6• US Preventive Services Task Force. Screening and behavioral counseling interventions in primary care to reduce alcohol misuse: recommendation statement. Ann Intern Med 2004; 140:554-556. On the basis of an extensive review, widespread implementation of SBI for adults in primary care was recommended.

7• Denny CH, Serdula MK, Holtzman D, Nelson DE. Physician advice about smoking and drinking: are U.S. adults being informed? Am J Prev Med 2003; 24:7174. In a multi-state telephone survey, only $23 \%$ of binge drinkers, who received a routine medical check-up in the previous year, reported that the doctor had discussed their drinking.

8 Seppa K, Aalto M, Raevaara L, Perakyla A. A brief intervention for risky drinking: analysis of videotaped consultations in primary health care. Drug Alcohol Rev 2004; 23:167-170.

$9 \quad$ Aalto M, Seppa K. Usefulness, length and content of alcohol-related discussions in primary health care: the exit poll survey. Alcohol Alcohol 2004; 39:532-535.

10 Beich A, Gannik D, Malterud K. Screening and brief intervention for excessive alcohol use: qualitative interview study of the experiences of general practitioners. BMJ 2002; 325:870.

11 Lock CA, Kaner EF, Heather N, et al. Changes in receptionists' attitudes towards involvement in a general practice-based trial of screening and brief alcohol intervention. Br J Gen Pract 2000; 50:111-115.

12 McCambridge J, Platts S, Whooley D, Strang J. Encouraging GP alcohol intervention: pilot study of change-orientated reflective listening (CORL). Alcohol Alcohol 2004; 39:146-149.

13• Kaner E, Lock C, Heather N, et al. Promoting brief alcohol intervention by nurses in primary care: a cluster randomised controlled trial. Patient Educ Couns 2003; 51:277-284. Training resulted in more nurses implementing SBI, and more patients per practice given SBI for at-risk drinking. However, more patients who had no alcohol problems received SBI by nurses who had been trained in these techniques, and so cost-benefits were comparable across the three groups.

14• Toll BA, Sobell LC, D’Arienzo J, et al. What do Internet-based alcohol treatment websites offer? Cyberpsychol Behav 2003; 6:581-584. Few treatmentrelated sites actually provided on-line treatment, and most of these applied 12-step facilitation. Few sites provided references or outcome data on the treatment that they recommended.

15• Copeland J, Martin G. Web-based interventions for substance use disorders: a qualitative review. J Subst Abuse Treat 2004; 26:109-116. The review found considerable interest among health professionals and the public for web-based alcohol interventions, but little evidence of efficacy. 
16•• Gregor MA, Shope JT, Blow FC, et al. Feasibility of using an interactive laptop program in the emergency department to prevent alcohol misuse among adolescents. Ann Emerg Med 2003; 42:276-284. The feasibility of using e-SBI with teenage patients (14-18 years) admitted for acute injury was demonstrated. The intervention was completed by $78 \%$ of eligible patients and was acceptable to users. 17 Monti PM, Colby SM, Barnett NP, et al. Brief intervention for harm reduction with alcohol-positive older adolescents in a hospital emergency department. J Consult Clin Psychol 1999; 67:989-994.

18• Kypri K, Saunders JB, Gallagher SJ. Acceptability of various brief intervention approaches for hazardous drinking among university students. Alcohol Alcohol 2003; 38:626-628. A probability sample of university students (82\% response) indicated a strong preference for e-SBI over practitioner-delivered SBI in the context of primary care.

19 Squires DD, Hester RK. Using technical innovations in clinical practice: the Drinker's Check-Up software program. J Clin Psychol 2004; 60:159-169.

20 Crawford MJ, Lowe PC, Greene L, Brookings C. The prevalence of excessive alcohol consumption and the acceptability of brief advice in a sexual health clinic: cross sectional survey. Sex Transm Infect 2004; 80:416-417.

21• Gerbert B, Berg-Smith S, Mancuso M, et al. Using innovative video doctor technology in primary care to deliver brief smoking and alcohol intervention. Health Promot Pract 2003; 4:249-261. High acceptability of a laptop-based intervention was shown among patients attending their GP but its efficacy was not reported.

22• Wild TC, Roberts AB, Cunningham J, et al. Alcohol problems and interest in self-help: a population study of Alberta adults. Can J Public Health 2004; 95:127132. In a probability sample of Canadians (65\% response), 22\% expressed interest in receiving self-help materials for their drinking.

23 Lieberman DZ. Determinants of satisfaction with an automated alcohol evaluation program. Cyberpsychol Behav 2003; 6:677-682.

24 Linke S, Brown A, Wallace P. Down your drink: a web-based intervention for people with excessive alcohol consumption. Alcohol Alcohol 2004; 39:29-32.

25•• Saitz R, Helmuth ED, Aromaa SE, et al. Web-based screening and brief intervention for the spectrum of alcohol problems. Prev Med 2004; 39:969-975. An impressively large study in which use of a website offering e-SBI was characterized. The site was used by a broad spectrum of age groups. It shows that a well designed site can reach large numbers of problem drinkers.

26•• Kypri K, Saunders JB, Williams SM, et al. Web-based screening and brief intervention for hazardous drinking: a double-blind randomized controlled trial. Addiction 2004; 99:1410-1417. The study is the first the authors know of that examines the efficacy of e-SBI in a primary care setting. Patients of a university health service who received e-SBI reduced their consumption and related problems by $20-30 \%$ over 6 months relative to controls.

27• Curry SJ, Ludman EJ, Grothaus LC, et al. A randomized trial of a brief primary-care-based intervention for reducing at-risk drinking practices. Health Psychol 2003; 22:156-165. Patients who received physician counselling plus telephone support fared better than controls. However, initial consumption was low, with only $5 \%$ consuming four or more drinks per day. Most participants accepted only one call from the counsellor.

28•• Currie SR, Clark S, Hodgins DC, et al. Randomized controlled trial of brief cognitive-behavioural interventions for insomnia in recovering alcoholics. Addiction 
2004; 99:1121-1132. Patients receiving five sessions of individual CBT, or a self-help manual plus five brief phone calls had improvements in sleep relative to controls, but there was no evidence that the intervention prevented relapse to drinking.

29• Walters ST, Woodall WG. Mailed feedback reduces consumption among moderate drinkers who are employed. Prev Sci 2003; 4:287-294. Employees receiving mailed personalized feedback decreased their consumption relative to controls. However, most participants were moderate drinkers and only $7 \%$ of potential respondents participated.

30•• Schinke SP, Schwinn TM, Di Noia J, Cole KC. Reducing the risks of alcohol use among urban youth: three-year effects of a computer-based intervention with and without parent involvement. J Stud Alcohol 2004; 65:443-449. A computerized primary prevention program assisting 10-12-year-olds to delay onset of drinking reduced alcohol use and delayed initiation to heavy drinking at 1, 2 and 3-year followups compared with controls.

31 - Murphy JG, Benson TA, Vuchinich RE, et al. A comparison of personalized feedback for college student drinkers delivered with and without a motivational interview. J Stud Alcohol 2004; 65:200-203. Heavy drinking psychology students receiving personal drinking feedback or personal drinking feedback plus a motivational interview did not differ significantly in their consumption 6 months later.

32• Neighbors C, Larimer ME, Lewis MA. Targeting misperceptions of descriptive drinking norms: efficacy of a computer-delivered personalized normative feedback intervention. J Consult Clin Psychol 2004; 72:434-447. Heavy drinking psychology students who received e-SBI reported significantly greater reductions in drinking at 3 and 6-month follow-up compared with controls.

33 Cunningham JA, Humphreys K, Koski-Jannes A. Providing personalized assessment feedback for problem drinking on the Internet: a pilot project. J Stud Alcohol 2000; 61:794-798.

34 Sitharthan T, Kavanagh DJ, Sayer G. Moderating drinking by correspondence: an evaluation of a new method of intervention. Addiction 1996; 91:345-355.

35 Richmond R, Kehoe L, Heather N, Wodak A. Evaluation of a workplace brief intervention for excessive alcohol consumption: the workscreen project. Prev Med 2000; 30:51-63.

36 Foxcroft DR, et al. Longer-term primary prevention for alcohol misuse in young people: a systematic review. In: Kettil Bruun Society Thematic Meeting 'Preventing Substance Use, Risky Use and Harm: What is Evidence Based Policy?'; Fremantle, Western Australia; 24-27 Feburary 2003.<AQ1>

37 Cunningham JA, Koski-Jannes A, Wild TC, et al. Treating alcohol problems with self-help materials: a population study. J Stud Alcohol 2002; 63:649-654.

Table 1 Acceptability of various alcohol-related services on campus

\begin{tabular}{|c|c|c|c|c|c|c|}
\hline & \multicolumn{2}{|c|}{$\begin{array}{c}\text { (A) It should be } \\
\text { available to students } \\
(n=1519)\end{array}$} & \multicolumn{2}{|c|}{$\begin{array}{l}\text { (B) I would use it if I } \\
\text { had a problem } \\
(n=1519)\end{array}$} & \multicolumn{2}{|c|}{$\begin{array}{l}\text { (B) I would use it if I } \\
\text { had a problem } \\
\text { (hazardous drinkers } \\
\text { only; } n=980 \text { ) }\end{array}$} \\
\hline & $n$ & $\%$ & $n$ & $\%$ & $n$ & $\%$ \\
\hline $\begin{array}{l}\text { Reading materials/leaflets about } \\
\text { alcohol and its effects }\end{array}$ & 1447 & 95.3 & 1110 & 73.1 & 690 & 72.6 \\
\hline $\begin{array}{l}\text { Health education seminars on } \\
\text { alcohol }\end{array}$ & 1244 & 81.9 & 626 & 41.2 & 381 & 40.1 \\
\hline Anonymous web-based alcohol & 1400 & 92.2 & 1232 & 81.1 & 778 & 81.9 \\
\hline
\end{tabular}




\begin{tabular}{|c|c|c|c|c|c|c|}
\hline \multicolumn{7}{|l|}{$\begin{array}{l}\text { risk assessment and } \\
\text { personalised feedback (e-SBI) }\end{array}$} \\
\hline $\begin{array}{l}\text { Alcohol risk assessment and } \\
\text { advice from a nurse, counsellor, } \\
\text { or psychologist (SBI) }\end{array}$ & 1374 & 90.5 & 932 & 61.4 & 551 & 58.0 \\
\hline $\begin{array}{l}\text { Alcohol risk assessment and } \\
\text { advice from a doctor (SBI) }\end{array}$ & 1332 & 87.7 & 921 & 60.6 & 551 & 58.0 \\
\hline
\end{tabular}

